\title{
Evaluating the Efficacy and Side Effects of Corticosteroid Therapy in Severely ill COVID-19 Patients
}

\section{Yun Cai}

Nanjing Medical University

\section{Lu Li}

Nanjing Medical University

Jing Chen

Zhejiang University School of Medicine, Zhejiang University

\section{Kening Li}

Nanjing Medical University

\section{Shenli Zhang}

Nanjing Medical University

\section{Ziyu Wang}

Nanjing Medical University

Jie Li

Nanjing Medical University

Mengyan Zhu

Nanjing Medical University

\section{Bin Huang}

Nanjing Medical University

\section{Lingxiang $\mathbf{W u}$}

Nanjing Medical University

\section{Wei Wu}

Nanjing Medical University

\section{Min Wu}

Nanjing Medical University

\section{Rong Ding}

Nanjing Medical University

\section{Xiuzhi Jia}

Zhejiang University

\section{Chaochen Wang}

Zhejiang University School of Medicine, Zhejiang University

\section{Qianghu Wang}


Nanjing Medical University

\section{Xinyi Xia}

Nanjing University School of Medicine

\section{Ying Xiao}

Zhejiang University

Sali Lyu ( $\square$ Ivsali@njmu.edu.cn )

Nanjing Medical University

\section{Research Article}

Keywords: COVID-19, Corticosteroid therapy, Side effect, Immune response

Posted Date: August 9th, 2021

DOI: https://doi.org/10.21203/rs.3.rs-620540/v1

License: (c) (i) This work is licensed under a Creative Commons Attribution 4.0 International License. Read Full License 


\section{Abstract}

Background: Cytokine storms are a common complication in severely ill patients with COVID-19, for which corticosteroid therapy (CsT) is used as adjuvant treatment. Therefore, we evaluated the efficacy and safety of CsT in patients with COVID-19.

Methods: A single-center, retrospective cohort study was conducted in 1,392 severely ill patients with COVID-19 from Wuhan Huoshenshan Hospital. Patients received at least one dose (1-2 $\mathrm{mg} \cdot \mathrm{kg}^{-1} \cdot \mathrm{day}^{-1}$ for 3-5 days) of methylprednisolone were divided into CsT group, whereas the rest were assigned into the non-CsT group.

Results: Of 1,392 patients, 116 were assigned to the CsT group and 1,226 to the non-CsT group. Patients in the CsT group showed comparable mortality rate $(1.8 \%$ vs. $1.2 \%, P>0.99)$ and viral clearance time (44.5 days vs. 46.0 days, $P=0.48$ ), but longer hospitalization time ( 21 days vs. 12 days, $P<0.001$ ) than those in non-CsT group. During CsT, the proportion of lymphocytes was lower $(14.7 \%$ vs. $18.5 \%, P=$ $0.01)$, while neutrophils was higher $(77.1 \%$ vs. $69.8 \%, P<0.001)$, than before treatment. The C-reactive protein (CRP) level was significantly lower after CsT ( $3.1 \mathrm{mg} / \mathrm{L}$ vs. $9.5 \mathrm{mg} / \mathrm{L}, P<0.001)$. Furthermore, indicators of liver function (gamma-glutamyl transferase [GGT], alanine aminotransferase [ALT], and aspartate aminotransferase [AST]) and cardiac function (brain natriuretic peptide [BNP], ahydroxybutyrate dehydrogenase [a-HBDH], and lactate dehydrogenase [LDH]) increased significantly during CsT but returned to normal after CsT. Patients who developed liver damage showed higher GGT, ALT, AST, LDH, Cre, and CRP; patients who developed heart injury had higher AST, LPH, CRP, lymphocyte (LYM), glucose, BNP, and a-HBDH; and patients who developed kidney failure had higher a-HBDH, LDH, CRP, and LYM values than before CsT. Additionally, patients who received CsT with cardiovascular disease showed a continuous elevation in D-dimer levels.

Conclusions: CsT effectively attenuates the inflammatory response in severely ill patients with COVID-19 at a relatively low dose in a short duration; however, CsT increases the risk of hepatic and cardiac abnormalities.

\section{Background}

Coronavirus disease 2019 (COVID-19), which is caused by severe acute respiratory syndrome coronavirus (SARS-CoV-2), has spread rapidly worldwide[1]. As of June 10, 2021, the ongoing COVID-19 pandemic has resulted in about 174,949,567 cases and 3,759,536 deaths globally (https://who.sprinklr.com/), and the number of deaths is still increasing. According to data from China, approximately $20 \%$ of patients with COVID-19 develop severe disease, which is characterized by the rapid development of severe acute respiratory distress syndrome (ARDS), septic shock, and multiple organ dysfunction syndrome [1, 2]. However, the pathogenesis of COVID-19 remains unclear and there is currently no effective therapy or vaccine available for this novel coronavirus. 
Cytokine storms are a common complication in severely ill patients with COVID-19. The excessive and uncontrolled release of cytokines leads to hyperinflammation, thereby resulting in acute lung injury, ARDS, and death $[3,4]$. However, this process can be dampened by anti-inflammatory drugs such as tocilizumab and glucocorticoids [5-7]. Glucocorticoids are widely used in autoimmune diseases, asthma, and lung diseases to suppress inappropriate inflammatory responses [8]; however, they are associated with a range of adverse events, such as hyperglycemia, avascular necrosis, and osteoporosis [9]. Corticosteroids, in other coronaviral pneumonias such as SARS and Middle East respiratory syndrome (MERS), have shown adverse reactions and side effects, especially when used at a high dosage for an extended period. For instance, hydrocortisone is administered at $300 \mathrm{mg} /$ day for a median duration of 7 days in critical MERS cases and at $100 \mathrm{mg}$ every 8 hours for 12 days in early SARS cases, thereby delaying coronavirus RNA clearance because of immunosuppression [11,21]. Moreover, an overdose of methylprednisolone in patients with SARS leads to the impairment of metabolic function, hyperglycemia, avascular necrosis, and osteoporosis [10,11]. The World Health Organization does not recommend the routine use of systemic corticosteroids; hence, glucocorticoid application has been restricted to a low dose $\left(1-2 \mathrm{mg} \cdot \mathrm{kg}^{-}\right.$ ${ }^{1} \cdot$ day $^{-1}$ ) for a short term (< 5 days) for the treatment of patients with severe COVID-19 in China [12]. Corticosteroid therapy (CsT) has been used as an adjuvant therapy under the Guidance released by China's National Health Commission (referred to as Guidance hereafter) for patients with COVID-19 who have progressed rapidly. Nevertheless, an evaluation of the efficacy and safety of this strategy would benefit clinicians worldwide who encounter severely ill patients with COVID-19.

Although one retrospective study has reported that corticosteroids decrease the risk of death in patients with ARDS [13], another has reported no benefits [14]. However, the efficacy, side effects, and outcomes of corticosteroid use in patients with COVID-19 are still unknown. Here, we retrospectively analyzed 1,392 severely ill patients with COVID-19 admitted to Wuhan Huoshenshan Hospital, China, from February 4 to March 30, 2020, to evaluate the efficacy and safety of methylprednisolone in COVID-19, expecting to present a valuable reference for the treatment of patients with COVID-19.

\section{Methods}

\section{Data collection}

This study was approved by the Medical Ethical Committee of Wuhan Huoshenshan Hospital. Written informed consent was obtained from each patient. This cohort included 1392 severely ill COVID-19 patients admitted from February 4 to March 30 to Wuhan Huoshenshan Hospital, which is one of the biggest hospitals designated for COVID-19 patients in Wuhan. all patients were treated with standard therapy recommended in the Diagnosis and Treatment Protocol for Novel Coronavirus Pneumonia (Version 6 or trial Version 7) released by the National Health Commission. The severity degree of the disease of each patient was determined according to clinical classification criteria described in this protocol. 
Among the 1392 cases, 166 patients accepted corticosteroid therapy (CsT). These patients were injected with at least one dose (1-2 $\mathrm{mg} \mathrm{kg}^{-1} \cdot \mathrm{day}^{-1}$ for 3-5 days) of methylprednisolone and included in the CsT group. Meanwhile, 1226 patients were not treated by CsT, these patients were included in the non-CsT group.

Data were extracted from patient records, including age, sex, comorbidities, time from symptom onset to hospital admission, vital signs at admission, laboratory testing results during hospitalization, SARS-CoV2 nucleic acid testing results, dose and time of treatment, and clinical outcome.

\section{Outcomes and definitions}

The primary outcomes were the cure rate and mortality rate. The secondary outcomes were the virus clearance time and hospitalization time. Virus clearance was confirmed by multiple tests of RT-PCR using throat swab specimens. Patients whose virus nuclei acid test results of respiratory tract specimens were negative twice consecutively (with at least a 24-hour sampling interval) were considered virus-free.

\section{Propensity score matching}

The propensity score is defined as the probability of each participant being assigned to a group of interest for comparison purposes, which was used to ensure an even distribution of confounders between groups [15].

\section{Evaluation of side effects for cardiac, hepatic and renal injury}

Serum markers of cardiac function, including B-type natriuretic peptide (BNP), a-hydroxybutyrate dehydrogenase ( $\mathrm{a}-\mathrm{HBDH})$, creatine kinase (CK), creatine kinase-MB (CK-MB), and lactic dehydrogenase $(\mathrm{LDH})$, were used to evaluate cardiac abnormalities in severely ill COVID-19 patients. Cardiac injury was defined as an elevated serum level for at least one of these indicators that above the normal reference limit. Patients with normal cardiac function before CsT were included in the comparison. Patients with cardiac abnormalities after CsT were defined as the injured group, and those without were defined as the normal group.

The same method was used to access hepatic and renal injury. Serum markers of hepatic function including alanine aminotransferase (ALT), aspartate aminotransferase (AST), $\gamma$-glutamyl transpeptidase (GGT), and alkaline phosphatase (ALP) were used. Serum markers of kidney function including creatinine (CR) and uric acid (UA) were used.

\section{Observation point selection}


Because there were multiple tests for each patient, data from various time points were selected according to different purposes of each analysis. To compare the patient's conditions in CsT and Non-CsT group when they admitted, we selected the first test result of each patients, and selected the last test result to compare their clinical outcomes. To analyze the dynamic changes of biochemical indicators among CsT patients, we described the average levels of the indicators at three stages: before CsT, during CsT, and after CsT. Only 159 cured patients were included in this analysis.

\section{Statistical analysis}

Continuous variables were summarized as medians with interquartile ranges (IQRs), and categorical variables were presented as frequencies and percentages. We used the Wilcoxon rank-sum test (2-sided) or Fisher's exact test to compare differences between groups, as appropriate. While counting the medians (Q1, Q4), the ratio of the detection results beyond the normal range was also counted. $P<0.05$ was considered statistically significant. All analyses were performed in R 3.6.2.

\section{Results}

\section{Characteristics of the cohort}

Of 3,059 patients diagnosed with COVID-19 in Wuhan Huoshenshan Hospital, mild and moderate cases, critically ill cases upon admission, and cases with unknown disease were excluded. In brief, 1,417 patients were identified as severely ill but 25 were excluded owing to previous treatment with prednisone acetate. Hence, 1,392 severely ill patients with COVID-19 were enrolled in this study for further analysis (Figure 1).

Among all selected patients, the median age was 63 years (IQR, 53-71), including 688 (49.4\%) men and 704 (50.6\%) women, indicating that there was no gender difference in the infection rate. Many patients had preexisting medical conditions, including hypertension (497 [35.7\%]), diabetes (221 [15.9\%]), and cardiovascular disease (211 [15.2\%]). The most common symptoms were fever (951 [68.3\%]), cough (829 [59.6\%]), and fatigue (544 [39.1\%]). Dyspnea (159 [11.4\%]) and chest pain (172 [12.4\%]) were also observed in some patients, whereas diarrhea (35 [2.5\%]), ache (8 [0.6\%]), and emesis (13 [0.9\%]) were fewer common symptoms (Table 1).

\section{Severely ill patients with COVID-19 receive CsT upon admission}

According to the severity degree of the disease progress during treatment, 166 patients $(11.9 \%)$ who received methylprednisolone intravenously as recommended by the Guidance were defined as the CsT group, whereas 1,226 patients who did not were defined as the non-CsT group. More patients in the CsT group had hypertension ( $42.2 \%$ vs. $34.8 \%, P=0.07)$, immunodeficiencies ( $1.8 \%$ vs. $0.2 \%, P=0.01)$, and 
acute respiratory distress ( $4.8 \%$ vs $0.2 \%, P<0.001)$, as well as other symptoms, including fatigue ( $48.2 \%$ vs. $37.9 \%, P=0.01)$, fever ( $76.5 \%$ vs. $67.2 \%, P=0.02)$, and dyspnea $(24.1 \%$ vs. $9.7 \%, P<0.001)$ than in the non-CsT group (Table 1). Additionally, the first laboratory tests after admission showed differences between the groups (Supplementary Table S1). The percentage of neutrophil (NEUT) (69.75 [62.0-80.2] \% vs. 63 [56.6-70.5] \%, $P<0.001$ ) was significantly higher, whereas the percentage of lymphocyte (LYM) $(18.5[11.8-27.1] \%$ vs. $25.9[19.3-32.0] \%, P<0.001)$ was significantly lower, in the CsT group than in the non-CsT group before treatment. These results indicate the wide spectrum of disease severity and support the use of methylprednisolone in severely ill patients with COVID-19.

To reduce bias caused by comorbidities, propensity score matching (PSM) was performed (Supplementary Table S2). In brief, 166 patients from the non-CsT group were selected for subsequent analysis after matching and the results of laboratory tests before CsT treatment were compared (Supplementary Table S3). There were significant differences in laboratory indicators, including C-reactive protein $(\mathrm{CRP})$, NEUT, and LYM $(P$ all $<0.01)$ and glucose $(\mathrm{GLU})$, D-dimer, total bilirubin, alkaline phosphatase, uric acid, and LDH $(P$ all $<0.05)$. However, there were no differences in the levels of mean platelet volume, platelet count (PLT), BNP, ALT, AST, and Cre between the groups. Additionally, more patients were admitted into the intensive care unit in the CsT group than in the non-CsT group. These data indicate that patients who received CsT had more serious medical conditions than those who did not receive CsT, even after PSM.

\section{Comparison of outcomes in CsT and non-CsT patients}

Although patients in the CsT group had more serious medical conditions, they recovered at a rate comparable with non-CsT patients $(95.8 \%$ vs. $95.2 \%, P>0.99)$ and there was no difference in viral clearance time between the CsT and non-CsT groups (44.5 [38.5-47.3] days vs. 46.0 [28.3-67.3] days, $P$ $=0.48$ ). Additionally, there was no difference in the mortality rate between the CsT and non-CsT groups $(1.8 \%$ vs. $1.2 \%$, respectively, $P>0.99)$ but the hospitalization time was longer in the CsT group than in the non-CsT group (21 [17-28] days vs. 12 [7-20] days, $P<0.001)$ (Supplementary Table S4). The recovery rate of patients in the non-CsT group after PSM (158/166 [95.2\%]) was similar to that before PSM $(1196 / 1226$ [97.6\%]) and there were no differences in serum indicators and outcomes between the groups regarding the final test results before discharge (Supplementary Table S5). These data suggest that CsT had significant clinical benefits for severely ill patients with COVID-19.

\section{CsT elicits an anti-inflammatory effect and may cause secondary infections}

To assess the anti-inflammatory effects of CsT, the levels of a variety of inflammatory indicators in patients before, during, and after CsT were compared. The CRP level decreased significantly after CsT (3.1 [1.2-8.2] mg/L vs. $9.5[2.8-40.6] \mathrm{mg} / \mathrm{L}$, after and before CsT, respectively, $P<0.001)$ but there was 
no significant difference in the CRP level in patients during CsT compared with before CsT (Table 2, Figure 2A). The proportion of NEUT was higher in patients during CsT than in patients before CsT (77.1 [68.9-84.3] \% vs. 69.8 [62.0-80.2] \%, during and before CsT, respectively, $P<0.001)$, whereas the proportion of LYM decreased significantly during treatment $(14.7[9.9-22.2] \%$ vs. $18.5[11.8-27.1] \%$, during and before CsT, respectively, $P=0.01$ ) (Table 2, Figure 2B-C). Four cases that presented this trend for CRP, NEUT and LYM are shown in Figure 3A-C. This analysis demonstrates the anti-inflammatory effects of intravenously administered methylprednisolone, with the associated compromise in immune function.

\section{CsT is associated with increased risk of metabolic, cardiac, hepatic, and kidney abnormalities}

To estimate the side effects of CsT, we compared laboratory indexes of patients before, during, and after CsT. To evaluate glucose metabolism, we compared the GLU level; $48.7 \%$ of patients were outside the normal range during treatment, whereas only $24.8 \%$ of patients were outside the normal range before treatment but most patients gradually returned to normal levels after CsT (5.1 [4.7-6.6] mmol/L vs. 5.9 [5.0-8.0] mmol/L, after and during CsT, respectively, $P<0.001$ ) (Figure 2D, Figure 3D).

We also investigated potential side effects of CsT that adversely affected heart, liver, and kidney function. The level of ALT, an indicator of hepatic function, increased significantly during CsT (32.8 [19.6-56.5] IU/L vs. 26.1 [17.2-44.1] IU/L, during and before CsT, respectively, $P=0.03$ ) (Table 2). The level of BNP, an indicator of cardiac function, also increased significantly during CsT (42.2 [0.0-100.0] pg/mL vs. 19.4 $[0.0-35.1] \mathrm{pg} / \mathrm{mL}$, during and before CsT, respectively, $P=0.05)$. The PLT, an indicator of infection, also increased during CsT (240.0 [188.0-309.0] × 109/L vs. 219 [161.3-284.0] × 109/L, during and before CsT, respectively, $P=0.01$ ).

To identify the patients most likely to suffer from cardiac, hepatic, and kidney abnormalities during CsT, we compared biochemical indicators between the injured and unaffected groups. In patients with hepatic abnormalities during CsT, the levels of ALT and GGT before CsT were significantly higher than those in unaffected patients during CsT (Table 3). Similarly, the levels of BNP, a-HBDH, and LDH before CsT were significantly higher in patients with cardiac abnormalities than in unaffected patients during CsT (Table 4). The levels of most biochemical indicators before CsT in patients with kidney abnormalities did not change significantly; only a-HBDH and LDH levels were higher than in unaffected patients, with an increase of CRP level and decrease of the proportion of LYM (Supplementary Table S6).

For patients with a common underlying disease, we conducted further research on the effect of CsT. Patients with hypertension had significantly increased PLT and ALT values during CsT (Supplementary Table S7) and patients with cardiovascular disease showed a continuous elevation in D-dimer level (Supplementary Table S8), whereas patients who had diabetes did not show excessive side effects during or after CsT (Supplementary Table S9). 


\section{Discussion}

In this study, we retrospectively reviewed 1,392 patients treated in Wuhan Huoshenshan Hospital and assessed the efficacy and safety of CsT in the treatment of severely ill patients with COVID-19. CsT was effective in the attenuation of the inflammatory response, with no obvious delay in virus elimination in severely ill patients with COVID-19.

Previous studies have reported that cytokine storms are common in severely ill patients with COVID-19, who present with significantly increased cytokine, such as interleukin-2 and tumor necrosis factor-alpha, levels $[16,17]$. A high concentration of glucocorticoids ( $>30 \mathrm{mg}$ prednisone equivalent a day) downregulates these cytokine levels via genomic and non-genomic actions [16] and is often used to prevent further injury. However, the clinical use of CsT in COVID-19 is still controversial. So far, studies have focused on the safety of CsT and dose and duration controls have been suggested to minimize systemic adverse reactions [9]. In the present study, we have provided further support that the inflammatory parameters in most patients return to normal levels after CsT at a low dose $\left(1-2 \mathrm{mg} \cdot \mathrm{kg}^{-}\right.$ ${ }^{1} \cdot$ day $^{-1}$ ) for one course (3-5 days), with no increased mortality and comparable viral clearance time; thus, glucocorticoids may effectively attenuate the inflammatory response in severely ill patients with COVID-19.

However, because of immunosuppression by glucocorticoids, inappropriate glucocorticoid use may delay viral clearance. Zheng et al. showed that, in severely ill patients with COVID-19, the duration of the virus is significantly longer in patients treated with glucocorticoids continuously for more than 10 days than in patients treated with glucocorticoids for less than 10 days [17], although there was no analysis based on the glucocorticoid dose used. Additionally, the duration of SARS-CoV-2 varies in specimens from different tissues; longest in stool and shortest in the upper respiratory tract [17-19]. Wölfel et al. showed that viruses in the throat replicate independently and are not shed from the lung [18], raising the possibility that the effects of glucocorticoids on viral clearance might be tissue-dependent; thus, the sampling site might affect the monitoring of viral clearance time. Nevertheless, the results of the present and a previous study show that CsT at relatively low dose $\left(1-2 \mathrm{mg} \mathrm{kg}^{-1} \cdot \mathrm{day}^{-1}\right)$ for less than 5 days does not delay virus elimination in severe patients [20], which indicates that a short period of glucocorticoid treatment at a low dose could be considered for the treatment of severely infected patients with COVID-19.

Although the regimen in our study effectively inhibited inflammation, transitory heart, liver, and kidney abnormalities were observed in some patients during CsT. It is important to use the shortest course possible and monitor liver and cardiac functions during CsT; thus, liver, cardiac, and kidney protective drugs can be applied, especially for patients with underlying diseases, such as cardiovascular diseases. The prevalence of cardiovascular complications, acute liver injury, and acute kidney injury is high among patients with COVID-19 who are severely or critically ill [20], which may be induced by the direct activity of the virus or an excessive immune response [21, 22]. 
In a cytokine storm, inflammation caused by the immune response can overwhelm the body and damage healthy organs such as the heart, liver, and kidneys. SARS-CoV-2 seems to have a preference for the vascular system because of the expression of the angiotensin-converting enzyme 2 receptor [23]. Furthermore, viral inclusion bodies have been observed in endothelial cells that are indispensable for vascular homeostasis and the regulation of multiple organ functions. As a result, inflammatory cells associated with endothelium accumulate in the lung, heart, liver, and kidneys, as well as intestine, which suggests that SARS-CoV-2 may induce endotheliitis in several organs as a direct consequence of viral and host inflammatory response involvement [23]. When we investigated the five deaths in our study cohort, three showed underlying diseases, such as hypertension, diabetes, or cerebrovascular disease, which affect the endothelium. However, whether virus-induced endotheliitis caused these deaths is uncertain.

Glucocorticoid receptors are expressed in most cells and operate in a context-specific manner. Acute exposure to glucocorticoids enhances, rather than suppresses, the peripheral immune response under stress conditions [24]. The present study showed that glucocorticoid use exaggerated pre-existing damage to the heart and liver. Taken together, virus-induced endothelial cell dysfunction and inflammation might have been amplified by glucocorticoids, which led to heart and liver injury. However, the pro-inflammatory effects of glucocorticoids might be temporary, as we observed large fluctuations in most biochemical indictors during the early stages of CsT, which returned to normal levels after CsT. Further studies are required to investigate the effects of glucocorticoids on virus-induced inflammation within specific cell types, such as endothelial cells.

Considering patients with a common underlying disease as a group, we studied the three deaths owing to an underlying disease separately rather than with the other patients. Of these three patients, one patient suffered from chronic obstructive pulmonary disease, one from hypertension, and one from hypertension, diabetes, and acute respiratory distress syndrome. We did not continue to study the effects of treatment on the deaths of these three patients because they all died just after CsT administration; thus, the effect of treatment on these deaths is not clear and a larger sample is needed in future research.

Several studies have suggested that CsT had benefited patients with COVID-19, especially severely ill patients. In a recent retrospective study of 201 patients, Wu et al. reported that corticosteroids reduce the rate of death in patients with COVID-19, pneumonia, and ARDS [13]. In another study, Zhou et al. recognized the benefit of low-dose CsT in a limited population of 15 severely ill patients with COVID-19, although there was no control group [25]. Wang et al. showed that a low-dose $\left(1-2 \mathrm{mg} \cdot \mathrm{kg}^{-1} \cdot \mathrm{day}^{-1}\right)$, short-term (3-5 days), early application corticosteroid is associated with better clinical outcomes in a small cohort of 46 severely ill patients with COVID-19 and suggested that this regimen should be considered before the onset of ARDS [26].

Our study has several advantages compared to these published reports. For example, our study included more cases than previous studies, as 1,392 severely ill patients with COVID-19 (166 CsT vs 1,226 nonCsT) were analyzed owing to the large number of patients that required treatment in Wuhan 
Huoshenshan Hospital. Moreover, we evaluated not only the anti-inflammatory effects but also the possible side effects of CsT, which provides useful implications for the treatment of COVID-19.

However, this study also has some limitations. Firstly, as each patient was monitored at different observation points, the presented data are the overall results of the group at each point. As representative examples, four individual patients with multiple observation points that showed the same trend as the group as a whole are presented. However, a more complete dataset, in which data for each patient are recorded at the same points, would provide better evidence for our conclusion. Secondly, after discharge, no prolonged adverse effects were analyzed. For a more accurate interpretation of the data, doubleblinded randomized clinical trials and follow-up visits should be conducted in the future. Thirdly, as all severe patients evaluated by the clinicians were administered CsT, this study lacks an ideal control severe group that did not receive CsT. Nevertheless, our analysis presents a valuable reference for the treatment of patients with COVID-19.

\section{Conclusions}

The administration of CsT at $1-2 \mathrm{mg} \cdot \mathrm{kg}^{-1} \cdot \mathrm{day}^{-1}$ for less than 5 days is effective in the attenuation of the inflammatory response in severely ill patients with COVID-19, with no risk of delaying viral clearance or increasing mortality. However, such a regimen could potentially exaggerate pre-existing injuries in the liver and heart. Thus, it is important to evaluate the medical condition of patients before the use of CsT and monitor these patients carefully during CsT treatment.

\section{Abbreviations}

Non-CsT: Patients without corticosteroid therapy; CsT: Patients received corticosteroid therapy; CRP: Creaction protein; NEUT: neutrophil; LYM: lymphocyte; GLU: glucose; MPV: mean platelet volume; PLT: platelet count; BNP: B-type natriuretic peptide; TBIL: total bilirubin; ALP: alkaline phosphatase; ALT: alanine aminotransferase; AST: Aspartate aminotransferase; UA: uric acid; Cre: creatinine; LDH: lactate dehydrogenase; GGT: y-glutamyl transpeptidase; a- $\mathrm{HBDH}$ : alpha-hydroxybutyric dehydrogenase; $\mathrm{CK}$ : Creatine kinase; $\mathrm{CK}-\mathrm{MB}$ : Creatine kinase-MB; $\mathrm{MCH}$ : Mean corpuscular hemoglobin.

\section{Declarations}

\section{Ethics approval and consent to participate}

This study was approved by the Medical Ethical Committee of Wuhan Huoshenshan Hospital (HSSLL011), and the Ethical Committee of Nanjing Medical University (2020-511).

\section{Consent for publication}

Not applicable 


\section{Availability of data and materials}

All data were collected from Wuhan Huoshenshan Hospital. Data from this study is available from the corresponding author upon reasonable request.

\section{Competing interests}

The authors declare no competing interests.

\section{Funding}

This study was supported by the National Natural Science Foundation of China (Grant Nos. 81572893, 81972358, 91959113), Key Foundation of Wuhan Huoshenshan Hospital (Grant No. 2020[18]), Key Research\& Development Program of Jiangsu Province (Grant Nos. BE2017733, BE2018713), Medical Innovation Project of Logistics Service (Grant No. 18JS005), Basic Research Program of Jiangsu Province (Grant No. BK20180036).

\section{Authors' contributions}

S.L.L., X.X., X.Y.X. had full access to all of the data in the study and takes responsibility for the integrity of the data and the accuracy of the data analysis. Y.C., L.L., J.C., K.N.L. contributed equally. Concept and design: S.L.L., X.X., and X.Y.X. Experiments and data collection: X.Y.X. Data analysis and interpretation: Y.C., L.L., K.N.L., S.L.Z., Z.Y.W., J.L., M.Y.Z., B.H., L.X.W., W.W., M.W., R.D., J.C., C.C.W., X.Z.J., Y.X. Drafting of the manuscript: Y.C., L.L., J.C., Y.X. All authors have read and approved the final manuscript.

\section{Acknowledgements}

We thank Tingting Zhang and Yuan Liang for their help with data analysis. We thank Yifei Cheng for professional suggestions on drafting of the manuscript.

\section{References}

1. Zhu N, Zhang D, Wang W, Li X, Yang B, Song J, Zhao X, Huang B, Shi W, Lu R et al: A Novel Coronavirus from Patients with Pneumonia in China, 2019. N Engl J Med 2020, 382(8):727-733.

2. Chen Y, Liu Q, Guo D: Emerging coronaviruses: Genome structure, replication, and pathogenesis. $J$ Med Virol 2020, 92(4):418-423.

3. Mehta P, McAuley DF, Brown M, Sanchez E, Tattersall RS, Manson JJ, Hlh Across Speciality Collaboration UK: COVID-19: consider cytokine storm syndromes and immunosuppression. Lancet 2020, 395(10229):1033-1034. 
4. Channappanavar R, Perlman S: Pathogenic human coronavirus infections: causes and consequences of cytokine storm and immunopathology. Semin Immunopathol 2017, 39(5):529539 .

5. Ai J, Li Y, Zhou X, Zhang W: COVID-19: treating and managing severe cases. Cell Res 2020, 30(5):370-371.

6. Chen N, Zhou M, Dong X, Qu J, Gong F, Han Y, Qiu Y, Wang J, Liu Y, Wei Y et al: Epidemiological and clinical characteristics of 99 cases of 2019 novel coronavirus pneumonia in Wuhan, China: a descriptive study. Lancet 2020, 395(10223):507-513.

7. Xu X, Han M, Li T, Sun W, Wang D, Fu B, Zhou Y, Zheng X, Yang Y, Li X et al: Effective treatment of severe COVID-19 patients with tocilizumab. Proc Natl Acad Sci U S A 2020.

8. Rice JB, White AG, Scarpati LM, Wan G, Nelson WW: Long-term Systemic Corticosteroid Exposure: A Systematic Literature Review. Clinical therapeutics 2017, 39(11):2216-2229.

9. Shah A, Mak D, Davies AM, James SL, Botchu R: Musculoskeletal Corticosteroid Administration: Current Concepts. Canadian Association of Radiologists journal = Journal l'Association canadienne des radiologistes 2019, 70(1):29-36.

10. Xiao JZ, Ma L, Gao J, Yang ZJ, Xing XY, Zhao HC, Jiao JS, Li GW: [Glucocorticoid-induced diabetes in severe acute respiratory syndrome: the impact of high dosage and duration of methylprednisolone therapy]. Zhonghua nei ke za zhi 2004, 43(3):179-182.

11. Weinstein RS: Glucocorticoid-induced osteoporosis and osteonecrosis. Endocrinology and metabolism clinics of North America 2012, 41(3):595-611.

12. Shang L, Zhao J, Hu Y, Du R, Cao B: On the use of corticosteroids for 2019-nCoV pneumonia. Lancet 2020, 395(10225):683-684.

13. Wu C, Chen X, Cai Y, Xia J, Zhou X, Xu S, Huang H, Zhang L, Zhou X, Du C et al: Risk Factors Associated With Acute Respiratory Distress Syndrome and Death in Patients With Coronavirus Disease 2019 Pneumonia in Wuhan, China. JAMA Intern Med 2020.

14. Wang D, Hu B, Hu C, Zhu F, Liu X, Zhang J, Wang B, Xiang H, Cheng Z, Xiong Y et al: Clinical Characteristics of 138 Hospitalized Patients With 2019 Novel Coronavirus-Infected Pneumonia in Wuhan, China. JAMA 2020.

15. Baek S, Park SH, Won E, Park YR, Kim HJ: Propensity score matching: a conceptual review for radiology researchers. Korean J Radiol 2015, 16(2):286-296.

16. Stahn C, Buttgereit F: Genomic and nongenomic effects of glucocorticoids. Nature clinical practice Rheumatology 2008, 4(10):525-533.

17. Zheng S, Fan J, Yu F, Feng B, Lou B, Zou Q, Xie G, Lin S, Wang R, Yang X et al: Viral load dynamics and disease severity in patients infected with SARS-CoV-2 in Zhejiang province, China, JanuaryMarch 2020: retrospective cohort study. BMJ (Clinical research ed) 2020, 369:m1443.

18. Wölfel R, Corman VM, Guggemos W, Seilmaier M, Zange S, Müller MA, Niemeyer D, Jones TC, Vollmar $\mathrm{P}$, Rothe $\mathrm{C}$ et al: Virological assessment of hospitalized patients with COVID-2019. Nature 2020, 581(7809):465-469. 
19. Ling Y, Xu SB, Lin YX, Tian D, Zhu ZQ, Dai FH, Wu F, Song ZG, Huang W, Chen J et al: Persistence and clearance of viral RNA in 2019 novel coronavirus disease rehabilitation patients. Chinese medical journal 2020, 133(9):1039-1043.

20. Fang X, Mei Q, Yang T, Li L, Wang Y, Tong F, Geng S, Pan A: Low-dose corticosteroid therapy does not delay viral clearance in patients with COVID-19. The Journal of infection 2020, 81(1):147-178.

21. Varga Z, Flammer AJ, Steiger P, Haberecker M, Andermatt R, Zinkernagel AS, Mehra MR, Schuepbach RA, Ruschitzka F, Moch H: Endothelial cell infection and endotheliitis in COVID-19. Lancet (London, England) 2020, 395(10234):1417-1418.

22. Cao X: COVID-19: immunopathology and its implications for therapy. Nature reviews Immunology 2020, 20(5):269-270.

23. Ferrario CM, Jessup J, Chappell MC, Averill DB, Brosnihan KB, Tallant EA, Diz DI, Gallagher PE: Effect of angiotensin-converting enzyme inhibition and angiotensin II receptor blockers on cardiac angiotensin-converting enzyme 2. Circulation 2005, 111(20):2605-2610.

24. Dhabhar FS: Stress-induced augmentation of immune function-the role of stress hormones, leukocyte trafficking, and cytokines. Brain, behavior, and immunity 2002, 16(6):785-798.

25. Zhou W, Liu Y, Tian D, Wang C, Wang S, Cheng J, Hu M, Fang M, Gao Y: Potential benefits of precise corticosteroids therapy for severe 2019-nCoV pneumonia. Signal Transduct Target Ther 2020, 5(1):18.

26. Wang Y, Jiang W, He Q, Wang C, Wang B, Zhou P, Dong N, Tong Q: A retrospective cohort study of methylprednisolone therapy in severe patients with COVID-19 pneumonia. Signal Transduct Target Ther 2020, 5(1):57.

\section{Tables}

\section{Table 1}

Comparison of baseline characteristics of severely ill COVID-19 patients between non-CsT and CsT group. 


\begin{tabular}{|c|c|c|c|c|}
\hline Characteristics & Total & Non-CsT & CsT & P Value \\
\hline Number of patients, No. (\%) & 1392 & $1226(88.1)$ & $166(11.9)$ & - \\
\hline Age (years), median (IQR) & $63(53-71)$ & $63(53-71)$ & $63(53.3-69)$ & 0.1 \\
\hline Sex & & & & 0.02 \\
\hline Men & 688 & $591(48.2)$ & $97(58.4)$ & - \\
\hline Women & 704 & $635(51.8)$ & $69(41.6)$ & - \\
\hline \multicolumn{5}{|l|}{ Comorbid conditions, No. (\%) } \\
\hline Hypertension & $497(35.7)$ & $427(34.8)$ & $70(42.2)$ & 0.07 \\
\hline Diabetes & $221(15.9)$ & $198(16.2)$ & $23(13.9)$ & 0.50 \\
\hline Cardiovascular disease & $211(15.2)$ & $188(15.3)$ & $23(13.9)$ & 0.72 \\
\hline Cerebrovascular disease & $78(5.6)$ & $70(5.7)$ & $8(4.8)$ & 0.86 \\
\hline Cancer & $54(3.9)$ & $49(4.0)$ & $5(3.0)$ & 0.67 \\
\hline Chronic kidney disease & $28(2.0)$ & $22(1.8)$ & $6(3.6)$ & 0.13 \\
\hline Chronic liver disease & $37(2.7)$ & $34(2.8)$ & $3(1.8)$ & 0.61 \\
\hline Immunodeficiency & $5(0.4)$ & $2(0.2)$ & $3(1.8)$ & 0.01 \\
\hline Acute respiratory distress & $10(0.7)$ & $2(0.2)$ & $8(4.8)$ & $<0.001$ \\
\hline \multicolumn{5}{|l|}{ Signs and symptoms, No. (\%) } \\
\hline Fatigue & $544(39.1)$ & $464(37.9)$ & $80(48.2)$ & 0.01 \\
\hline Fever & $951(68.3)$ & $824(67.2)$ & $127(76.5)$ & 0.02 \\
\hline Cough & $829(59.6)$ & $727(59.3)$ & $102(61.5)$ & 0.61 \\
\hline Dyspnea & $159(11.4)$ & $119(9.7)$ & $40(24.1)$ & $<0.001$ \\
\hline Chest pain & $172(12.4)$ & $145(11.8)$ & $27(16.3)$ & 0.10 \\
\hline Ache & $8(0.6)$ & $7(0.6)$ & $1(0.6)$ & $<0.001$ \\
\hline Emesis & $13(0.9)$ & $10(0.8)$ & $3(1.8)$ & 0.20 \\
\hline Diarrhea & $35(2.5)$ & $32(2.6)$ & $3(1.8)$ & 0.79 \\
\hline Rhizobium & $3(0.2)$ & $3(0.2)$ & $0(0)$ & $>0.99$ \\
\hline Headache & $11(0.8)$ & $9(0.7)$ & $2(1.2)$ & 0.63 \\
\hline Pharyngalgia & $2(0.1)$ & $2(0.2)$ & $0(0)$ & $>0.99$ \\
\hline Hematemesis & $2(0.1)$ & $1(0.1)$ & $1(0.6)$ & 0.22 \\
\hline
\end{tabular}




\begin{tabular}{|lcccc} 
Chills & $0(0)$ & $0(0)$ & $0(0)$ & $>0.99$ \\
Non-CsT: Patients without corticosteroid therapy; CsT: Patients received corticosteroid therapy.
\end{tabular}

\section{Table 2}

The dynamics of various biochemical indicators in CsT group $(n=159)$ before, during, and after CsT. 


\begin{tabular}{|c|c|c|c|c|c|c|c|}
\hline & $\begin{array}{l}\text { Reference } \\
\text { range }\end{array}$ & $\begin{array}{l}\text { Before } \\
\text { CsT }\end{array}$ & $\begin{array}{l}\text { During } \\
\text { CsT }\end{array}$ & $\begin{array}{l}\text { After } \\
\text { CsT }\end{array}$ & $\begin{array}{l}\text { P value } \\
\text { during vs } \\
\text { before }\end{array}$ & $\begin{array}{l}\text { P value } \\
\text { after vs } \\
\text { during }\end{array}$ & $\begin{array}{l}\text { P value } \\
\text { after vs } \\
\text { before }\end{array}$ \\
\hline \multirow{2}{*}{$\begin{array}{l}\text { CRP** } \\
\text { (mg/L) }\end{array}$} & \multirow[t]{2}{*}{$0-4$} & 9.5 & 8.7 & 3.1 & \multirow[t]{2}{*}{0.65} & \multirow[t]{2}{*}{$<0.001$} & \multirow[t]{2}{*}{$<0.001$} \\
\hline & & $\begin{array}{l}(2.8- \\
40.7)\end{array}$ & $\begin{array}{l}(1.8- \\
39.0)\end{array}$ & $(1.2-8.2)$ & & & \\
\hline \multirow{2}{*}{$\begin{array}{l}\text { NEUT** } \\
(\%)\end{array}$} & \multirow[t]{2}{*}{$40-75$} & 69.8 & 77.1 & 68.8 & \multirow[t]{2}{*}{$<0.001$} & \multirow[t]{2}{*}{$<0.001$} & \multirow[t]{2}{*}{0.26} \\
\hline & & $\begin{array}{l}(62.0- \\
80.2)\end{array}$ & $\begin{array}{l}(68.9- \\
84.3)\end{array}$ & $\begin{array}{l}(62.5- \\
76.4)\end{array}$ & & & \\
\hline \multirow[t]{2}{*}{ LYM* (\%) } & \multirow[t]{2}{*}{$20-50$} & 18.5 & 14.7 & 20.8 & \multirow[t]{2}{*}{0.01} & \multirow[t]{2}{*}{$<0.001$} & \multirow[t]{2}{*}{0.12} \\
\hline & & $\begin{array}{l}(11.8- \\
27.1)\end{array}$ & $\begin{array}{l}(9.9- \\
22.2)\end{array}$ & $\begin{array}{l}(14.3- \\
26.4)\end{array}$ & & & \\
\hline \multirow{2}{*}{$\begin{array}{l}\text { GLU** } \\
(\mathrm{mmol} / \mathrm{L})\end{array}$} & \multirow[t]{2}{*}{$3.9-6.1$} & 5.22 & 5.9 & 5.1 & \multirow[t]{2}{*}{$<0.001$} & \multirow[t]{2}{*}{$<0.001$} & \multirow[t]{2}{*}{0.65} \\
\hline & & $(4.8-5.8)$ & $(5-8.0)$ & $(4.7-6.6)$ & & & \\
\hline \multirow[t]{2}{*}{ MPV (fL) } & \multirow[t]{2}{*}{$8.4-12.6$} & 9.9 & 10 & 9.9 & \multirow[t]{2}{*}{0.63} & \multirow[t]{2}{*}{0.62} & \multirow[t]{2}{*}{0.98} \\
\hline & & $\begin{array}{l}(9.2- \\
10.6)\end{array}$ & $\begin{array}{l}(9.3- \\
10.6)\end{array}$ & $\begin{array}{l}\text { (9.1- } \\
10.7)\end{array}$ & & & \\
\hline \multirow{2}{*}{$\begin{array}{l}\text { PLT* } \\
(\times 109 / L)\end{array}$} & \multirow[t]{2}{*}{$125-350$} & 219 & 240 & 222 & \multirow[t]{2}{*}{0.01} & \multirow[t]{2}{*}{0.01} & \multirow[t]{2}{*}{0.38} \\
\hline & & $\begin{array}{l}(161.3- \\
284)\end{array}$ & $\begin{array}{l}(188- \\
309)\end{array}$ & $\begin{array}{l}(179- \\
269.5)\end{array}$ & & & \\
\hline \multirow{2}{*}{$\begin{array}{l}\text { BNP } \\
(\mathrm{pg} / \mathrm{ml})\end{array}$} & \multirow[t]{2}{*}{$0-100$} & 19.4 & 42.2 & 14.7 & \multirow[t]{2}{*}{0.05} & 0.00 & 0.60 \\
\hline & & $\begin{array}{l}(0.0- \\
35.1)\end{array}$ & $\begin{array}{l}(0.0- \\
100.0)\end{array}$ & $\begin{array}{l}(0.0- \\
49.6)\end{array}$ & & & \\
\hline D-Dimer & $0-0.6$ & 0.8 & 0.9 & 0.8 & 0.11 & 0.06 & 0.99 \\
\hline & & $(0.4-1.8)$ & $(0.6-2.1)$ & $(0.5-1.3)$ & & & \\
\hline TBIL* & $0-26$ & 10 & 8.9 & 9.5 & 0.01 & 0.02 & 0.37 \\
\hline & & $\begin{array}{l}(7.9- \\
12.3)\end{array}$ & $(6.6-11)$ & $\begin{array}{l}(7.4- \\
12.3)\end{array}$ & & & \\
\hline ALP (IU/L) & $45-125$ & 65.6 & 67.4 & 71.2 & 0.60 & 0.44 & 0.15 \\
\hline & & $\begin{array}{l}(56.3- \\
83.1)\end{array}$ & $\begin{array}{l}(55.6- \\
84.7)\end{array}$ & $\begin{array}{l}(60- \\
83.5)\end{array}$ & & & \\
\hline ALT (IU/L) & $7-40$ & 26.1 & 32.8 & 32.1 & 0.03 & 0.40 & 0.06 \\
\hline & & $\begin{array}{l}(17.2- \\
44.1)\end{array}$ & $\begin{array}{l}(19.6- \\
56.5)\end{array}$ & $\begin{array}{l}(19.8- \\
52.7)\end{array}$ & & & \\
\hline AST (IU/L) & $7-45$ & 22.2 & 25.1 & 19.2 & 0.29 & $<0.001$ & $<0.001$ \\
\hline
\end{tabular}




\begin{tabular}{|c|c|c|c|c|c|c|c|}
\hline & & $\begin{array}{l}(17.8- \\
31.6)\end{array}$ & $\begin{array}{l}(17.9- \\
35.5)\end{array}$ & $\begin{array}{l}(15.3- \\
25.8)\end{array}$ & & & \\
\hline \multirow{2}{*}{$\begin{array}{l}\text { UA } \\
\text { (umol/L) }\end{array}$} & \multirow[t]{2}{*}{$202-416$} & 267 & 231.5 & 252 & \multirow[t]{2}{*}{0.05} & \multirow[t]{2}{*}{0.04} & \multirow[t]{2}{*}{0.68} \\
\hline & & $\begin{array}{l}(192- \\
306)\end{array}$ & $\begin{array}{l}(175- \\
301.5)\end{array}$ & $\begin{array}{l}(196- \\
305)\end{array}$ & & & \\
\hline \multirow{2}{*}{$\begin{array}{l}\text { Cre } \\
\text { (umol/L) }\end{array}$} & \multirow[t]{2}{*}{$57-111$} & 69.9 & 65.3 & 66.3 & \multirow[t]{2}{*}{0.03} & \multirow[t]{2}{*}{0.80} & \multirow[t]{2}{*}{0.01} \\
\hline & & $\begin{array}{l}(58.7- \\
86.4)\end{array}$ & $\begin{array}{l}(55.4- \\
80.0)\end{array}$ & $\begin{array}{l}(54.2- \\
75.7)\end{array}$ & & & \\
\hline \multirow[t]{2}{*}{$\mathrm{LDH}(\mathrm{IU} / \mathrm{L})$} & \multirow[t]{2}{*}{$120-250$} & 230.2 & 243.4 & 199.5 & \multirow[t]{2}{*}{0.07} & \multirow[t]{2}{*}{$<0.001$} & \multirow[t]{2}{*}{$<0.001$} \\
\hline & & $\begin{array}{l}(194.3- \\
282.7)\end{array}$ & $\begin{array}{l}(204.6- \\
306.0)\end{array}$ & $\begin{array}{l}(176.2- \\
223.3)\end{array}$ & & & \\
\hline \multicolumn{8}{|c|}{$\begin{array}{l}\text { CRP: C-reaction protein; NEUT: neutrophil; LYM: lymphocyte; GLU: glucose; MPV: mean platelet } \\
\text { volume; PLT: platelet count; BNP: B-type natriuretic peptide; TBIL: total bilirubin; ALP: alkaline } \\
\text { phosphatase; ALT: alanine aminotransferase; AST: Aspartate aminotransferase; UA: uric acid; Cre: } \\
\text { creatinine; LDH: lactate dehydrogenase; }\end{array}$} \\
\hline \multicolumn{8}{|c|}{ Before CsT: The test results of patients in CsT before treatment; } \\
\hline \multicolumn{8}{|c|}{ During CsT: The test results of patients in CsT during treatment; } \\
\hline \multicolumn{8}{|c|}{ After CsT: The test results of patients in CsT after treatment; } \\
\hline \multicolumn{8}{|c|}{$\star *: P$ value (during vs before) $<=0.001 ; \mathrm{P}$ value (during vs before) $<=0.01$} \\
\hline
\end{tabular}

\section{Table 3}

Comparison of biochemical indicators upon admission between normal and hepatic injured patients. 


\begin{tabular}{|c|c|c|c|c|}
\hline & Reference range & Normal patients $(n=35)$ & Injured patients $(n=54)$ & P Value \\
\hline GGT (IU/L) ** & $7-45$ & $25.1(20.4-32.1)$ & $40.5(33.3-77.6)$ & $<0.001$ \\
\hline $\operatorname{ALT}(\mathrm{IU} / \mathrm{L}) * \star$ & $7-40$ & $20.7(17.1-26.2)$ & $35.4(17.9-67.7)$ & $<0.001$ \\
\hline ALP (IU/L) & $45-125$ & $62.6(53.8-74.7)$ & $67.5(59.3-89.2)$ & 0.07 \\
\hline AST $(I U / L) * *$ & $7-45$ & $18.7(16.2-22.4)$ & $26.2(19.8-46.4)$ & $<0.001$ \\
\hline $\mathrm{BNP}(\mathrm{pg} / \mathrm{ml})$ & $0-100$ & $21.7(0.0-42.2)$ & $18.4(0.0-33.8)$ & 0.92 \\
\hline a- HBDH (IU/L) & $72-182$ & $178.5(151.3-207.8)$ & $202.2(157.3-249)$ & 0.02 \\
\hline CK (IU/L) & $40-200$ & $41.7(30.2-60.3)$ & $41.7(30.1-70.6)$ & 0.68 \\
\hline CK-MB (IU/L) & $0-24$ & $9.8(7.4-12.2)$ & $8.9(7.4-12.3)$ & 0.66 \\
\hline $\mathrm{LDH}(\mathrm{IU} / \mathrm{L})$ * & $120-250$ & $210.4(182.1-253.3)$ & $242.5(205.1-294.7)$ & 0.005 \\
\hline Cre (umol/L) ** & $57-111$ & $64.1(51.4-75.6)$ & $74.5(61.8-93.8)$ & 0.001 \\
\hline UA (umol/L) & $202-416$ & $276(195.8-328.3)$ & 263 (179.5-296.3) & 0.23 \\
\hline $\mathrm{CRP}(\mathrm{mg} / \mathrm{L}) * \star$ & $0-4$ & $4.8(1.5-12.9)$ & $13.7(5.1-64.0)$ & $<0.001$ \\
\hline PCT $(\mathrm{ng} / \mathrm{ml}) \star \star$ & $0-0.1$ & $0.0(0.0-0.1)$ & $0.1(0.1-0.1)$ & $<0.001$ \\
\hline NEUT (\%) & $40-75$ & $66.4(57-76)$ & $73.5(63.3-80.9)$ & 0.02 \\
\hline LYM (\%) & $20-50$ & $21.5(14.2-31.6)$ & $15.7(11.0-24.7)$ & 0.03 \\
\hline $\mathrm{MCH}(\mathrm{pg})$ & $27-34$ & $31.6(30.9-32.4)$ & $31(29.9-31.9)$ & 0.03 \\
\hline $\mathrm{GLU}(\mathrm{mmol} / \mathrm{L})$ * & 3.9-6.1 & $5.0(4.7-5.3)$ & $5.4(5.0-6.4)$ & 0.002 \\
\hline \multicolumn{5}{|c|}{$\begin{array}{l}\text { GGT: y-glutamyl transpeptidase; ALT: alanine aminotransferase; ALP: alkaline phosphatase; AST: } \\
\text { Aspartate aminotransferase; BNP: B-type natriuretic peptide; a- HBDH: alpha-hydroxybutyric } \\
\text { dehydrogenase; CK: Creatine kinase; CK-MB: Creatine kinase-MB; LDH: lactate dehydrogenase; Cre: } \\
\text { creatinine; UA: uric acid; CRP: C-reaction protein; PCT: Procalcitonin; NEUT: neutrophil; LYM: } \\
\text { lymphocyte; MCH: Mean corpuscular hemoglobin; GLU: glucose; }\end{array}$} \\
\hline \multicolumn{5}{|c|}{ Normal patients: patients whose hepatic function were keep normal after CsT; } \\
\hline \multicolumn{5}{|c|}{ Injured patients: patients whose hepatic function were abnormal after CsT; } \\
\hline \multicolumn{5}{|c|}{ Data used are the first laboratory testing results of patients after admission; } \\
\hline \multicolumn{5}{|c|}{$\star *$ : $P$ value $<=0.001 ; P$ value $<=0.01$} \\
\hline
\end{tabular}

\section{Table 4}


Comparison of biochemical indicators upon admission between normal and cardiac injured patients.

\begin{tabular}{|c|c|c|c|c|}
\hline & Reference range & Normal patients $(n=19)$ & Injured patients $(n=66)$ & $P$ Value \\
\hline GGT (IU/L) & $7-45$ & $35.3(25.5-58.8)$ & $34.5(24.3-55.9)$ & 0.84 \\
\hline ALT (IU/L) & $7-40$ & $21.1(15.7-28.6)$ & $28.5(17.5-49.8)$ & 0.05 \\
\hline ALP (IU/L) & $45-125$ & $76(60.5-96.6)$ & $64.2(55.6-79.7)$ & 0.05 \\
\hline AST (IU/L) ** & $7-45$ & $17.4(15.4-20.4)$ & $23.2(18.6-33.9)$ & $<0.001$ \\
\hline $\mathrm{BNP}(\mathrm{pg} / \mathrm{ml})$ * & $0-100$ & $0.0(0.0-10.3)$ & $25.4(11.3-57.0)$ & 0.004 \\
\hline $\mathrm{a}-\mathrm{HBDH}(\mathrm{IU} / \mathrm{L})$ ** & $72-182$ & $142.2(130.5-156.8)$ & 203.1 (175.5-247.3) & $<0.001$ \\
\hline CK (IU/L) & $40-200$ & $50.2(35.3-60.5)$ & $40.2(28.7-69.4)$ & 0.29 \\
\hline CK-MB (IU/L) & $0-24$ & $9.3(7.9-11.9)$ & $9.05(7.2-12.5)$ & 0.81 \\
\hline $\mathrm{LDH}(\mathrm{IU} / \mathrm{L})$ ** & $120-250$ & 175.9 (153.9-193) & 243.3 (211.5-292.8) & $<0.001$ \\
\hline Cre (umol/L) & $57-111$ & $76.8(61.1-86.7)$ & $68.9(57.9-85.8)$ & 0.41 \\
\hline UA (umol/L) & $202-416$ & $283(215.5-331)$ & $264.5(176.8-300.5)$ & 0.07 \\
\hline $\mathrm{CRP}(\mathrm{mg} / \mathrm{L}) * \star$ & $0-4$ & $1.8(1.2-4.1)$ & $12.8(4.77-49.09)$ & $<0.001$ \\
\hline РCT (ng/ml) & $0-0.1$ & $0.0(0.0-0.1)$ & $0.1(0.0-0.1)$ & 0.21 \\
\hline NEUT (\%) ** & $40-75$ & $64.5(56.3-69.6)$ & $71.3(62.6-81.3)$ & 0.001 \\
\hline LYM (\%) ** & $20-50$ & $24.4(19.3-33.4)$ & $16.4(10.9-24.8)$ & $<0.001$ \\
\hline $\mathrm{MCH}(\mathrm{pg})$ & $27-34$ & $31.4(30.4-32.3)$ & $31.2(30-32)$ & 0.46 \\
\hline $\mathrm{GLU}(\mathrm{mmol} / \mathrm{L})$ * & 3.9-6.1 & $4.8(4.5-5.7)$ & $5.3(5.0-5.8)$ & 0.01 \\
\hline \multicolumn{5}{|c|}{$\begin{array}{l}\text { GGT: y-glutamyl transpeptidase; ALT: alanine aminotransferase; ALP: alkaline phosphatase; AST: } \\
\text { Aspartate aminotransferase; BNP: B-type natriuretic peptide; a- HBDH: alpha-hydroxybutyric } \\
\text { dehydrogenase; CK: Creatine kinase; CK-MB: Creatine kinase-MB; LDH: Iactate dehydrogenase; Cre: } \\
\text { creatinine; UA: uric acid; CRP: C-reaction protein; PCT: Procalcitonin; NEUT: neutrophil; LYM: } \\
\text { lymphocyte; MCH: Mean corpuscular hemoglobin; GLU: glucose; }\end{array}$} \\
\hline \multicolumn{5}{|c|}{ Normal patients: patients whose cardiac function were keep normal after CsT; } \\
\hline \multicolumn{5}{|c|}{ Injured patients: patients whose cardiac function were abnormal after CsT; } \\
\hline \multicolumn{5}{|c|}{ Data used are the first laboratory testing results of patients after admission; } \\
\hline$\star *$ : $P$ value $<=0.0$ & value $<=0.01$ & & & \\
\hline
\end{tabular}

\section{Figures}




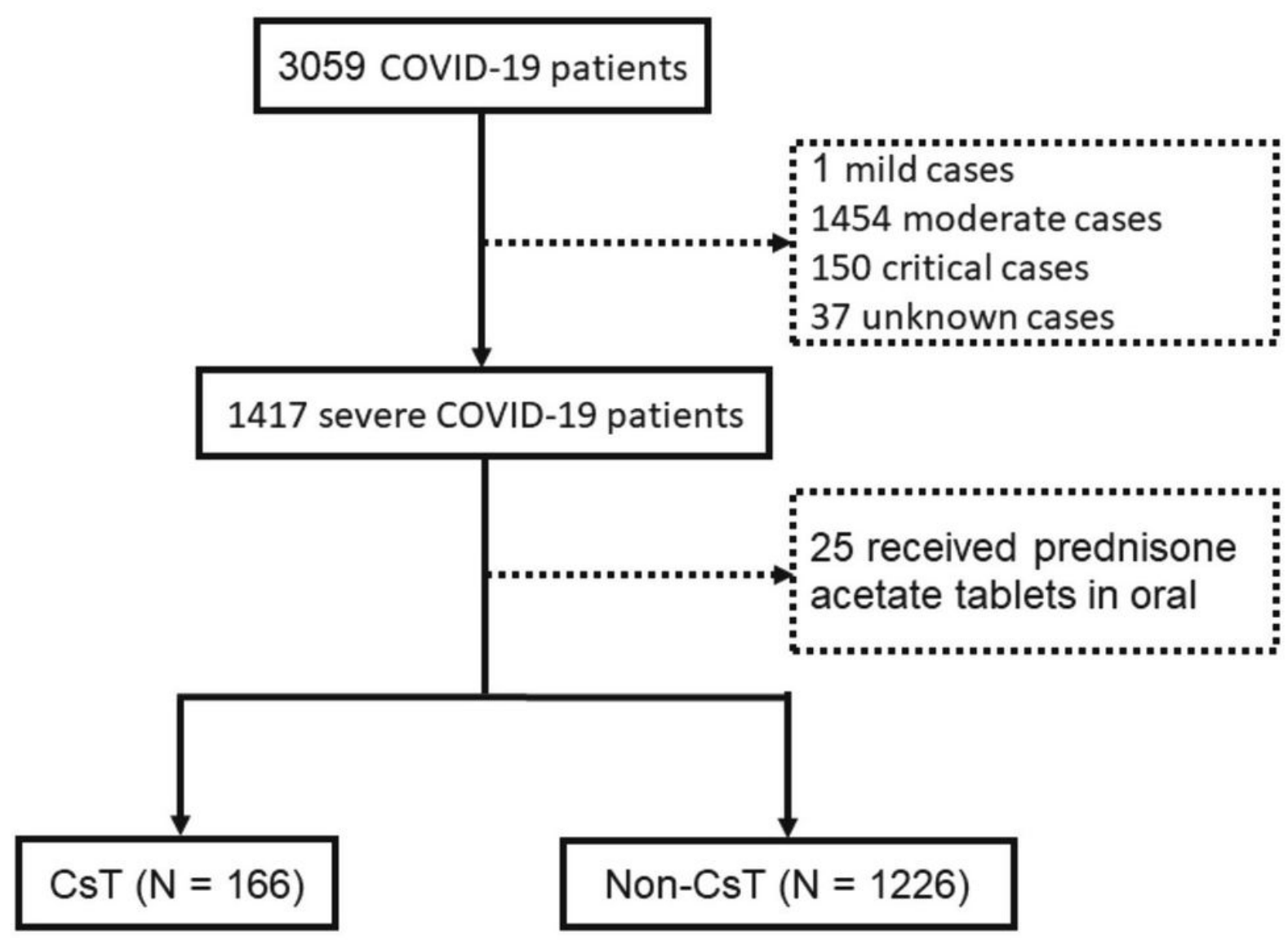

Figure 1

Flow chart of the patient selection. 3059 COVID-19 patients from a single center were screened, 1417 severely ill patients were selected, 25 patients received prednisone acetate tablets in oral were then removed for ensuring consistent mode of administration. At last, 166 patients received CsT and 1226 without CsT were included in this cohort study. 
A
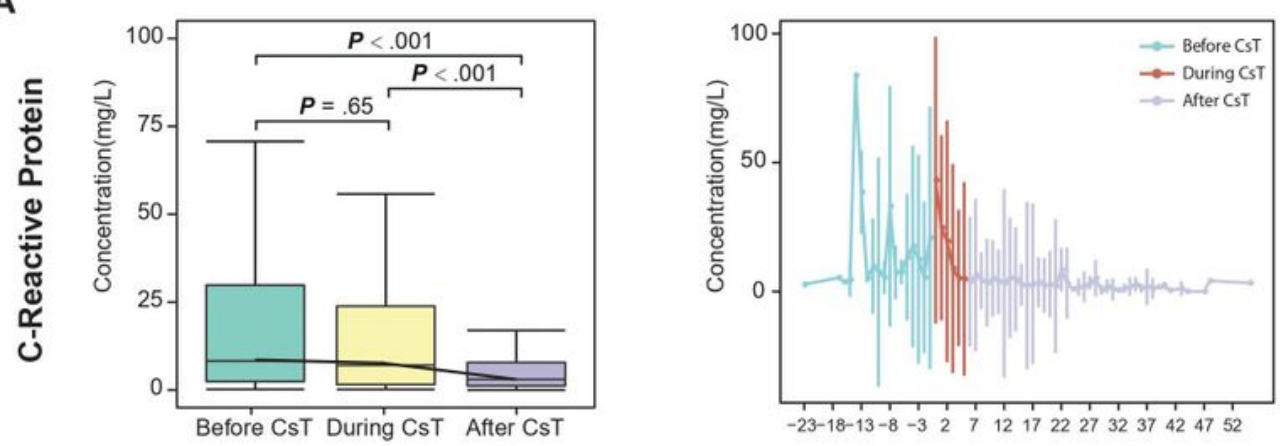

B
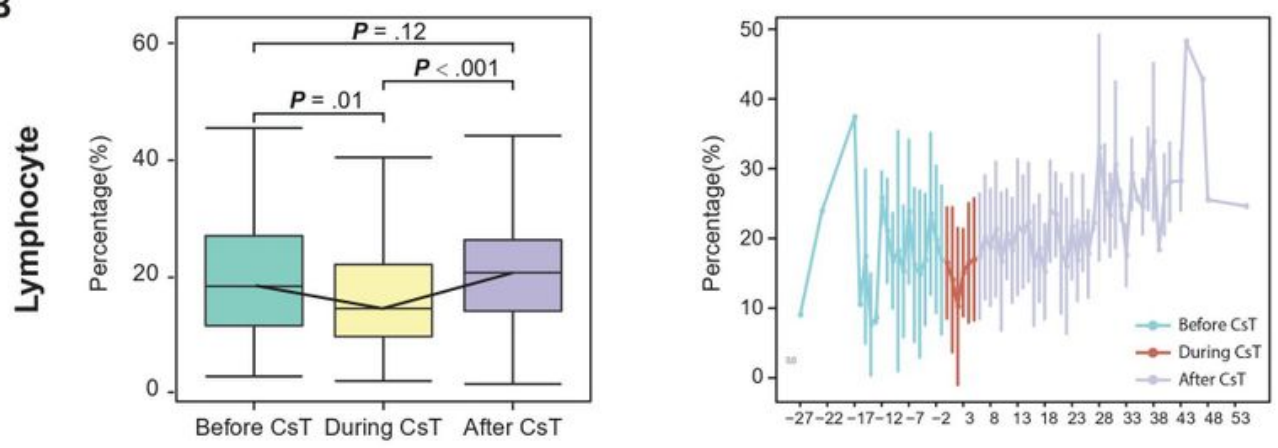

C
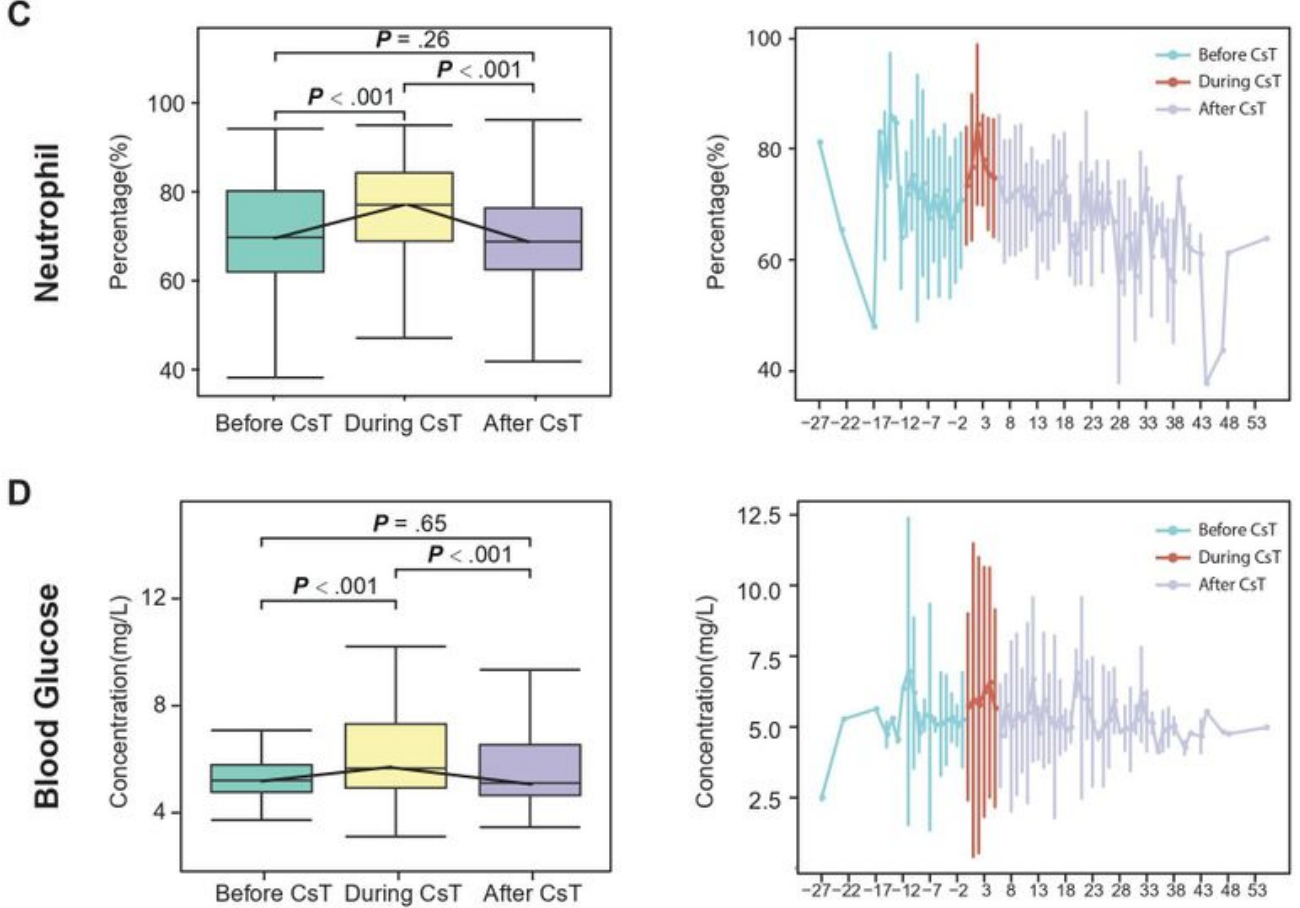

\section{Figure 2}

Comparison of the levels of laboratory results in patients among before, during and after CsT. The dynamics of (A) C-Reactive protein (CRP) concentration, the percentages of (B) lymphocyte (LYM) and (C) neutrophil (NEUT), (D) blood glucose (GLU) concentration of COVID-19 patients were showed. Figures on the left shows the variation tendency of laboratory results in patients before, during and after CsT. Figures on the right shows the dynamics of laboratory results in patients received CsT at a range of time intervals 
displayed as days. Data represented as the range of testing results (mean+/-SD). The $x$-axis of figures on the right displays the days from when CsT was given, zero of $\mathrm{x}$-axis represented the first time of CsT, and $y$-axis displays the level of testing results. Before CsT: The test results of patients in CsT group before treatment; During CsT: The test results of patients in CsT group during treatment; After CsT: The test results of patients in CsT group after treatment.

A
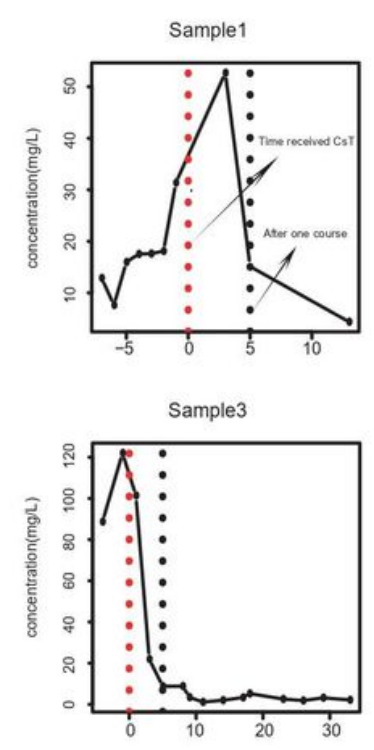

C
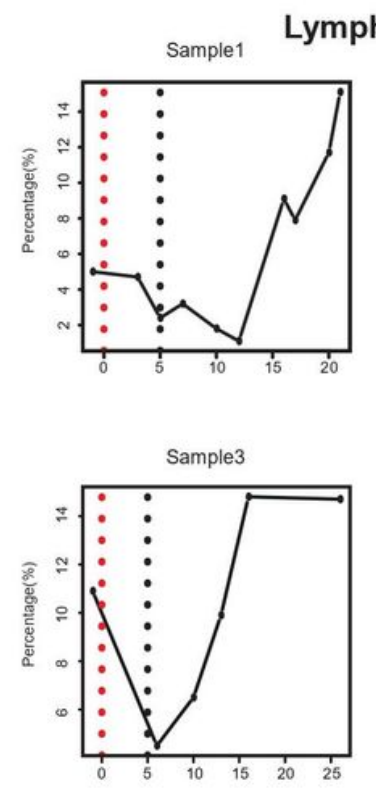
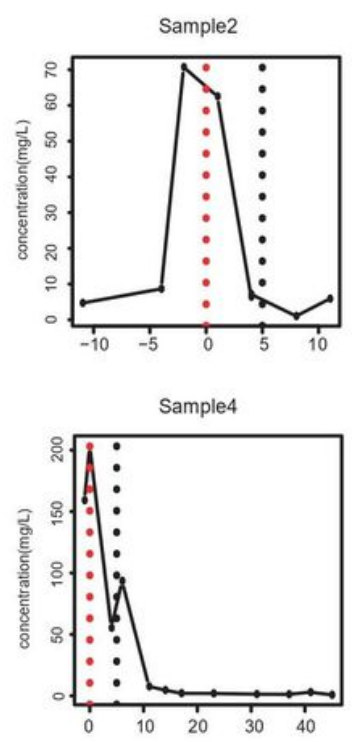

ymphocyte
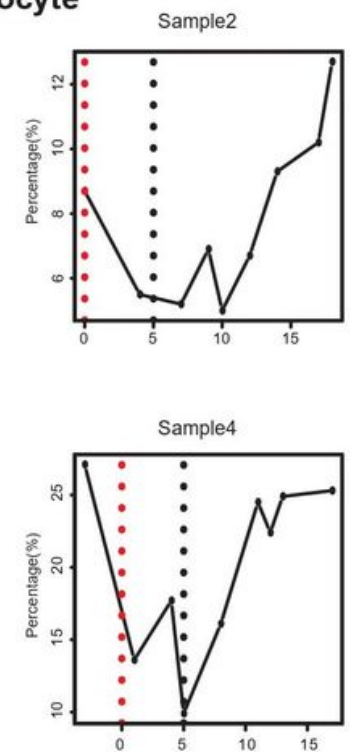

B

Neutrophil
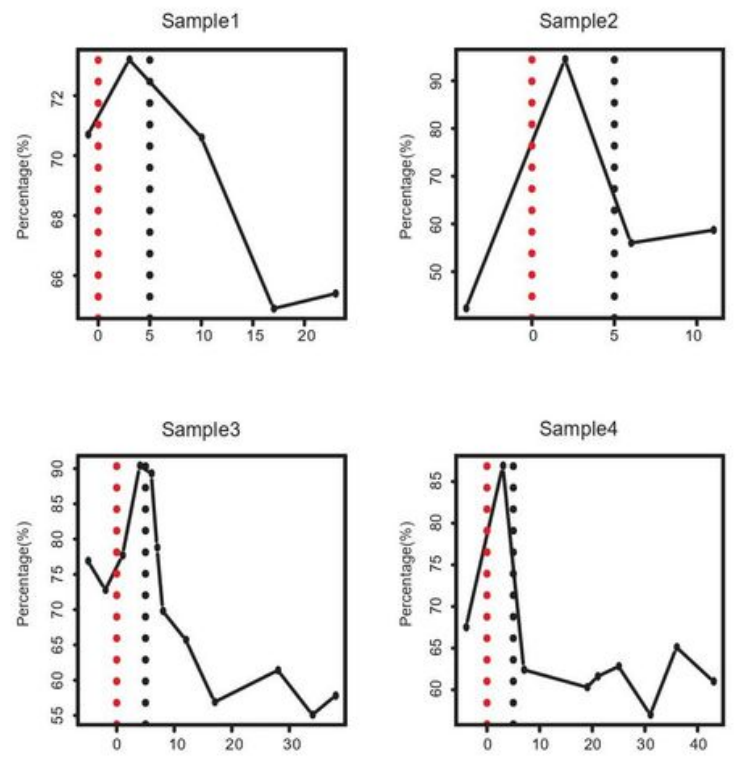

Blood Glucose

D
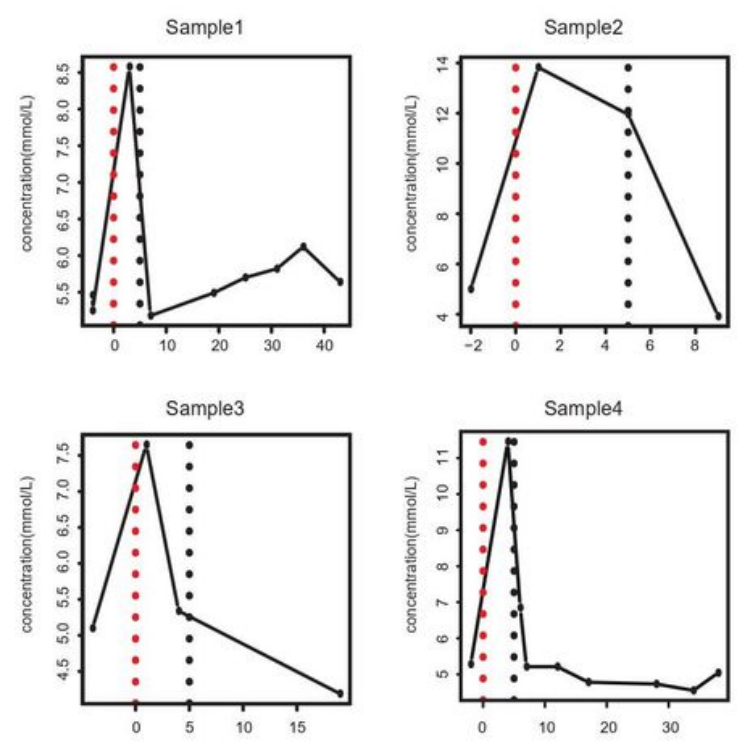

Figure 3 
Representative data of the dynamics of laboratory results in severely ill COVID-19 patients received CsT. The $x$-axis represents the days after admission, and y-axis represents the levels of each indicator. Red dash line indicates the day when first time received CsT. Black dash line indicates five days (one course of CsT) after first CsT. The dynamics change of (A) C-Reactive protein, (B) neutrophil percentage, (C) lymphocyte percentage, and (D) blood glucose concentration in four COVID-19 patients while hospitalized are presented.

\section{Supplementary Files}

This is a list of supplementary files associated with this preprint. Click to download.

- SupplementaryTables.docx 